\title{
EFFECT OF THE THICKNESS TO LENGTH RATIO ON THE FREQUENCY RATIO OF NANOBEAMS AND NANOPLATES
}

\author{
Arian Bahrami, Hamidreza Shiri, Nima Khosravi \\ Eastern Mediterranean University, Department of Mechanical Engineering,Magosa, TRNC Mersin, Turkey \\ e-mail: arianbahrami@ut.ac.ir
}

\begin{abstract}
This communication presents the effect of thickness on the frequency ratio of nanobeams and nanoplates using Eringen's nonlocal theory. Although there exist numerous works regarding the effects of thickness and small scale on the frequency ratio of nanobeams and nanoplates, none has captured and reported the true effects. The main intention of this communication is to correct the misunderstanding regarding this issue. It was found that the frequency ratio is indeed dependent on the thickness to length ratio and its variation with respect to thickness to length ratio is highly dependent on the mode number, combination of boundary conditions, plate aspect ratio, and the nonlocal parameter.
\end{abstract}

Keywords: nanobeam, nanoplate, nonlocal elasticity, frequency ratio, thickness

\section{Introduction}

Structures such as plates, beams, rods, shells and membranes are widely used in the engineering science at different scales (macro, micro and nano) (Bahrami et al., 2015; Bahrami and Teimourian, 2015a, 2017b; Sarayi et al., 2018; Zargaripoor and Bahrami, 2018). With the advent of nanotechnology, these structures are started to be used in micro/nanoelectromechanical systems (MEMS/NEMS), such in the form of electrically actuated MEMS devices (Zhang and Fu, 2012), atomic force microscopes (AFMs) (Kahrobaiyan et al., 2010), and etc.

Recently, several non-classical theories such as couple stress theory (Mindlin and Tiersten, 1962), modified couple stress theory (Yang et al., 2002), strain gradient theory (Mindlin, 1964), nonlocal elasticity theory (Eringen, 1972), and nonlocal strain gradient theory (Lim et al., 2015) try to capture the size effect in the structures. The scientific literature includes many papers based on different non-classical theories on the mechanical analysis of micro- and nano-sized structures such as micro/nanorod (Bahrami, 2017b), micro/nanobeam (Bahrami and Teimourian, 2015b, 2016; Dehrouyeh-Semnani and Bahrami, 2016; Arefi and Zenkour, 2017c; Bahrami, 2017a), and micro/nanoplates (Arefi and Zenkour, 2016, 2017a, 2017b; Ilkhani et al., 2016; Arefi et al., 2018; Bahrami and Teimourian, 2017a; Zenkour and Arefi, 2017).

While there are many papers in the literature regarding the effects of thickness and small scale on the frequency ratio of nanobeams and nanoplates using Eringen's nonlocal theory, unfortunately, most papers presented the thickness effect on the frequency ratio for fully simply supported cases due to simplicity of the calculation in this case (Aghababaei and Reddy, 2009; Aydogdu, 2009; Bahrami and Teimourian, 2016; Daneshmehr et al., 2015; Eltaher et al., 2012; Natarajan et al., 2012; Rahmani and Pedram, 2014; Reddy, 2007; Thai, 2012). As a result, the true effect of the thickness to length ratio on the frequency ratio of nanobeams and nanoplates was not captured and reported at all. As an attempt to demonstrate the effect for other combinations of boundary conditions, Hosseini-Hashemi et al. (2013) presented an exact solution to free vibration of Mindlin rectangular nanoplates using nonlocal first-order shear deformation plate theory. The authors claimed that for fixed values of the plate aspect ratio and the nonlocal 
parameter, the frequency ratio of a specific mode and a determined boundary condition remains unchanged throughout variations of the thickness to length ratio as shown in their Fig. 8 and stated clearly in page 298 and in the conclusion. Although the same reported frequency values can be obtained exactly according to the paper, the authors made a mistake in reporting this behavior. In another publication by Hosseini-Hashemi et al. (2015), the authors presented an exact solution to the buckling and free vibration of rectangular nanoplates using Reddy's nonlocal third-order shear deformation plate theory. Although the same reported frequency values can be obtained exactly according to the paper by Hosseini-Hashemi et al. (2015), the authors reported the same untrue behavior again. They concluded again that for fixed values of the aspect ratio and the nonlocal parameter, the frequency ratio of a specific mode and a determined boundary condition remains unchanged throughout variations of the thickness to length ratio as shown in their Fig. 4 and Table 10. To the best of the authors' knowledge, there is no literature presenting the true effect of the thickness to length ratio on the frequency ratio of nanobeams and nanoplates. As a result, the intention of this communication is to present the true effect of the thickness to length ratio on the frequency ratio of nanobeams and nanoplates and finally correct the misunderstanding among researchers once and for all.

\section{Nonlocal elasticity theory}

Among the non-classical theories, the nonlocal elasticity theory introduced by Eringen (1972) is one of the most popular non-classical continuum theories in which the effects of small scales are considered in the characteristic equations of this theory. In the classical theories of continuum mechanics, the stress in a point is related only to the strain in that specific point; however, in the nonlocal elasticity theory, the stress is also dependent on the strains at all points in the domain. In nonlocal elasticity theory, constitutive equations incorporate the effects of atomic forces and small scale as material parameters (Eringen, 2002). The differential form of the nonlocal constitutive equation has been developed by Eringen (2002) as follows

$$
\left[1-\left(e_{0} a\right)^{2} \nabla^{2}\right] \boldsymbol{\sigma}=\mathbf{C}: \boldsymbol{\varepsilon}
$$

where $\boldsymbol{\sigma}$ and $\varepsilon$ are the stress and strain tensors, $\mathbf{C}$ is the fourth order elastic modulus tensor, $\nabla^{2}$ indicates the Laplacian operator, $a$ indicates the internal length scale and $e_{0}$ is a physical parameter that has been identified by experimental results. The value of the parameter $a$ depends on the lattice parameter, granular size and C-C bonds, and the material constant. The parameter $\mu=\left(e_{0} a\right)^{2}$ is named the nonlocal parameter. Comparison of the continuum modeling results with those of atomistic ones determines the value of this parameter in any type of analysis.

\subsection{Mathematical formulation of the nanoplate}

Consider a thick rectangular nanoplate of length $a$, width $b$, and uniform thickness $h$ as shown in Fig. 1, oriented so that its undeformed middle surface contains the $x_{1}$ and $x_{2}$ axis of a cartesian coordinate system $\left(x_{1}, x_{2}, x_{3}\right)$, as shown in Fig. 1.

The displacements along the $x_{1}$ and $x_{2}$ axes are denoted by $U_{1}$ and $U_{2}$, respectively, while the displacement in the direction perpendicular to the undeformed middle surface is denoted by $U_{3}$. In the Mindlin plate theory, the displacement components are given as

$$
\begin{aligned}
& U_{1}\left(x_{1}, x_{2}, x_{3}, t\right)=u_{1}\left(x_{1}, x_{2}, t\right)+x_{3} \phi_{1}\left(x_{1}, x_{2}, t\right) \\
& U_{2}\left(x_{1}, x_{2}, x_{3}, t\right)=u_{2}\left(x_{1}, x_{2}, t\right)+x_{3} \phi_{2}\left(x_{1}, x_{2}, t\right) \\
& U_{3}\left(x_{1}, x_{2}, x_{3}, t\right)=u_{3}\left(x_{1}, x_{2}, t\right)
\end{aligned}
$$




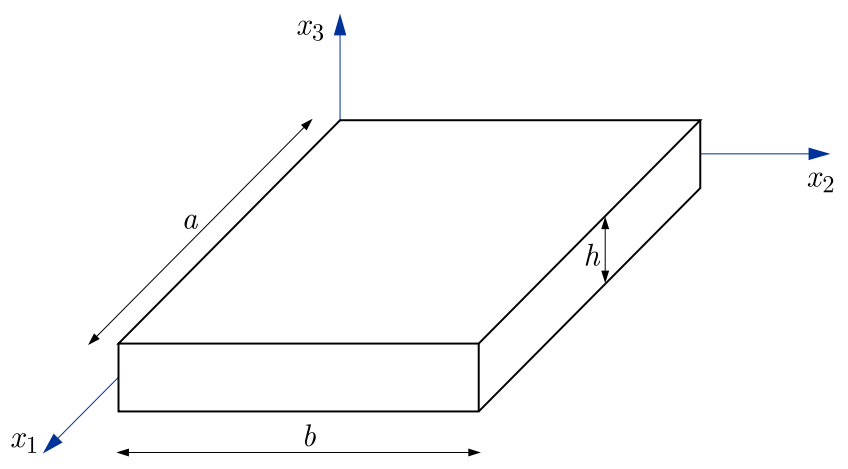

Fig. 1. A rectangular plate with coordinate convention

where $t$ is time, $u_{1}$ and $u_{2}$ are inplane displacements of points on the mid-plane (i.e. $x_{3}=0$ ), $u_{3}$ is the transverse displacement and $\phi_{i}(i=1,2)$ are slopes due to bending alone in the respective planes. The equations of motions of the Mindlin plate theory in the nonlocal continuum model for in-plane and out-of-plate displacements are uncoupled (Hosseini-Hashemi et al., 2013), so, for simplicity, we consider the equation of motion for flexural vibration. The non-dimensional form of equations of motion of the Mindlin plate theory in the nonlocal continuum model can be written as follows (Hosseini-Hashemi et al., 2013)

$$
\begin{aligned}
& \left(12 k \nu_{1}-\zeta^{2} \beta^{2} \delta^{2}\right) \widetilde{\nabla}^{2} \widetilde{u}_{3}+\beta^{2} \delta^{2} \widetilde{u}_{3}+12 k \nu_{1}\left(\widetilde{\phi}_{1,1}+\widetilde{\phi}_{2,2}\right)=0 \\
& \left(\nu_{1}-\frac{\zeta^{2} \beta^{2} \delta^{2}}{12}\right) \widetilde{\nabla}^{2} \widetilde{\phi}_{1}+\left(\frac{\beta^{2} \delta^{2}}{12}-\frac{12 k \nu_{1}}{\delta^{2}}\right) \widetilde{\phi}_{1}+\left(1-\nu_{1}\right)\left(\widetilde{\phi}_{1,11}+\widetilde{\phi}_{2,21}\right)-\frac{12 k \nu_{1}}{\delta^{2}} \widetilde{u}_{3,1}=0 \\
& \left(\nu_{1}-\frac{\zeta^{2} \beta^{2} \delta^{2}}{12}\right) \widetilde{\nabla}^{2} \widetilde{\phi}_{2}+\left(\frac{\beta^{2} \delta^{2}}{12}-\frac{12 k \nu_{1}}{\delta^{2}}\right) \widetilde{\phi}_{2}+\left(1-\nu_{1}\right)\left(\widetilde{\phi}_{1,12}+\widetilde{\phi}_{2,22}\right)-\frac{12 k \nu_{1}}{\delta^{2}} \widetilde{u}_{3,2}=0
\end{aligned}
$$

in which the non-dimensional Laplacian operator is defined as $\widetilde{\nabla}^{2}=\frac{\partial^{2}}{\partial X_{1}^{2}}+\frac{\partial^{2}}{\partial X_{2}^{2}}$ and the non-dimensional terms are considered as follows

$$
\begin{array}{lllr}
X_{i}=\frac{x_{i}}{a} & \delta=\frac{h}{a} & \eta=\frac{b}{a} \quad \widetilde{\phi}_{i}=\phi_{i} \quad i=1,2 \\
\widetilde{u}_{3}=\frac{u_{3}}{a} & \zeta=\frac{\sqrt{\mu}}{a} & \widetilde{\nabla}^{2}=a^{2} \nabla^{2} \quad \beta=\omega a^{2} \sqrt{\frac{\rho h}{D}}
\end{array}
$$

In these equations, $\zeta$ and $\beta$ are non-dimensional nonlocal and frequency parameters, respectively, $\mu$ is the nonlocal parameter, $\nu$ is Poisson's ratio and $\nu_{1}=(1-\nu) / 2$. The shear correction factor $k$ was introduced to consider that for the transverse shear strains, $D=E h^{3} /\left[12\left(1-\nu^{2}\right)\right]$ is the flexural rigidity.

The detailed solution of Eqs. (2.3) can be found in Hosseini-Hashemi et al. (2013). It is also possible to obtain a non-dimensional form of equations of motion based on the third-order shear deformation plate theory in the nonlocal continuum model. The detailed derivation and the solution can be found in Hosseini-Hashemi et al. (2015) in order to calculate the non-dimensional frequency parameter $\beta$. The frequency ratio (FR) of the nanoplate is defined as the ratio of the nonlocal frequency parameter to the local frequency parameter as

$$
(\mathrm{FR})=\frac{\beta_{e_{0} a}}{\beta_{e_{0} a=0}}
$$

\subsection{Mathematical formulation of the nanobeam}

The displacement fields of the elementary Timoshenko beams can be considered as

$$
u=z \phi(x, t) \quad w=w(x, t)
$$


where $(u, w)$ are the axial and transverse displacements of the point $(x, 0)$ on the mid-plane (i.e., $z=0), z$ is the coordinate measured from the mid-plane in the height direction of the beam and $\phi$ denotes rotation of the cross-section. The below governing nonlocal equation of a nonlocal Timoshenko beam can be obtained, see Bahrami and Teimourian (2016)

$$
\begin{aligned}
& G A k_{s}\left(\frac{\partial \phi}{\partial x}+\frac{\partial^{2} w}{\partial x^{2}}\right)=\rho A\left(\frac{\partial^{2} w}{\partial t^{2}}-\left(e_{0} a\right)^{2} \frac{\partial^{4} w}{\partial x^{2} \partial t^{2}}\right) \\
& E I \frac{\partial^{2} \phi}{\partial x^{2}}-G A k_{s}\left(\phi+\frac{\partial w}{\partial x}\right)=\rho I\left(\frac{\partial^{2} \phi}{\partial t^{2}}-\left(e_{0} a\right)^{2} \frac{\partial^{4} \phi}{\partial x^{2} \partial t^{2}}\right)
\end{aligned}
$$

in which $A$ is the cross-sectional area of the beam, $I$ is the moment of inertia of the beam, $\rho$ is mass density of the beam material, $E$ is the Young modulus elasticity, $G$ is the shear modulus, and $k_{s}$ is the shear correction in the Timoshenko beam theory. The detailed solution of Eqs. (2.7) can be found in Bahrami and Teimourian (2016) in order to calculate the non-dimensional frequency parameter $\beta=\omega L^{2} \sqrt{\rho A /(E I)}$ in which $L$ is length of the nanobeam. The frequency ratio (FR) of the nanobeam is defined as the ratio of the nonlocal frequency parameter to the local frequency parameter as

$$
(\mathrm{FR})=\frac{\beta_{e_{0} a}}{\beta_{e_{0} a=0}}
$$

\section{Results and discussion}

\subsection{Nanoplate results}

In this Section, the effect of the thickness to length ratio $\delta$ on the frequency ratio (FR) of nanoplates is presented in detail. Table 1 presents the frequency ratio (FR) (nonlocal frequency/local frequency) for different modes $(m, n)$, nonlocal parameters and thickness to length ratios using the nonlocal first-order shear deformation plate theory by Hosseini-Hashemi et al. (2013) in the case of SCSC boundary condition, and for $\eta=b / a=0.5$ as shown in their Fig. 8 (Hosseini-Hashemi et al., 2013). It is seen form Table 1 that the frequency ratio increases with an increase in the thickness to the length ratio for a fixed value of the nonlocal parameter. As the nonlocal parameter $\zeta$ increases, the difference in the frequency ratio increases and it reaches about $9 \%$ in the case of $\zeta=0.6$ which contradicts with their statement, and it should have been visible in their Fig. 8 as shown in our Fig. 2. This behavior has not been reported before by the nonlocal first-order shear deformation theory. Note that the frequency ratio difference increases as the mode number increases as presented in Table 1.

In order to verify such behavior, the calculation has been repeated again using a higher order theory, namely Reddy's nonlocal third-order shear deformation plate theory (Hosseini-Hashemi et al., 2015).

Table 2 presents the fundamental frequency ratio (FR) for different nonlocal parameters and thickness to length ratios using Reddy's nonlocal third-order shear deformation plate theory by Hosseini-Hashemi et al. (2015) in the case of SCSC boundary condition for three different aspect ratios $a / b=0.5,1,2$ as shown in their Fig. 4 and Table 10 (Hosseini-Hashemi et al., 2015). It is seen from Table 2 that the frequency ratio increases with an increase in the thickness to length ratio for a fixed value of the nonlocal parameter. As the nonlocal parameter $\zeta=\sqrt{\mu} / a$ increases, the difference in the fundamental frequency ratio increases and it reaches about $5 \%$ in the case of $\zeta=0.6, a / b=0.5$. This behavior has not been reported before by third-order shear deformation theories. It can be observed from their Table 10 by Hosseini-Hashemi et al. (2015) that their reported values of the frequency ratios remain unchanged throughout variations of thickness to length for all the mentioned boundary conditions. Such behavior is not correct 


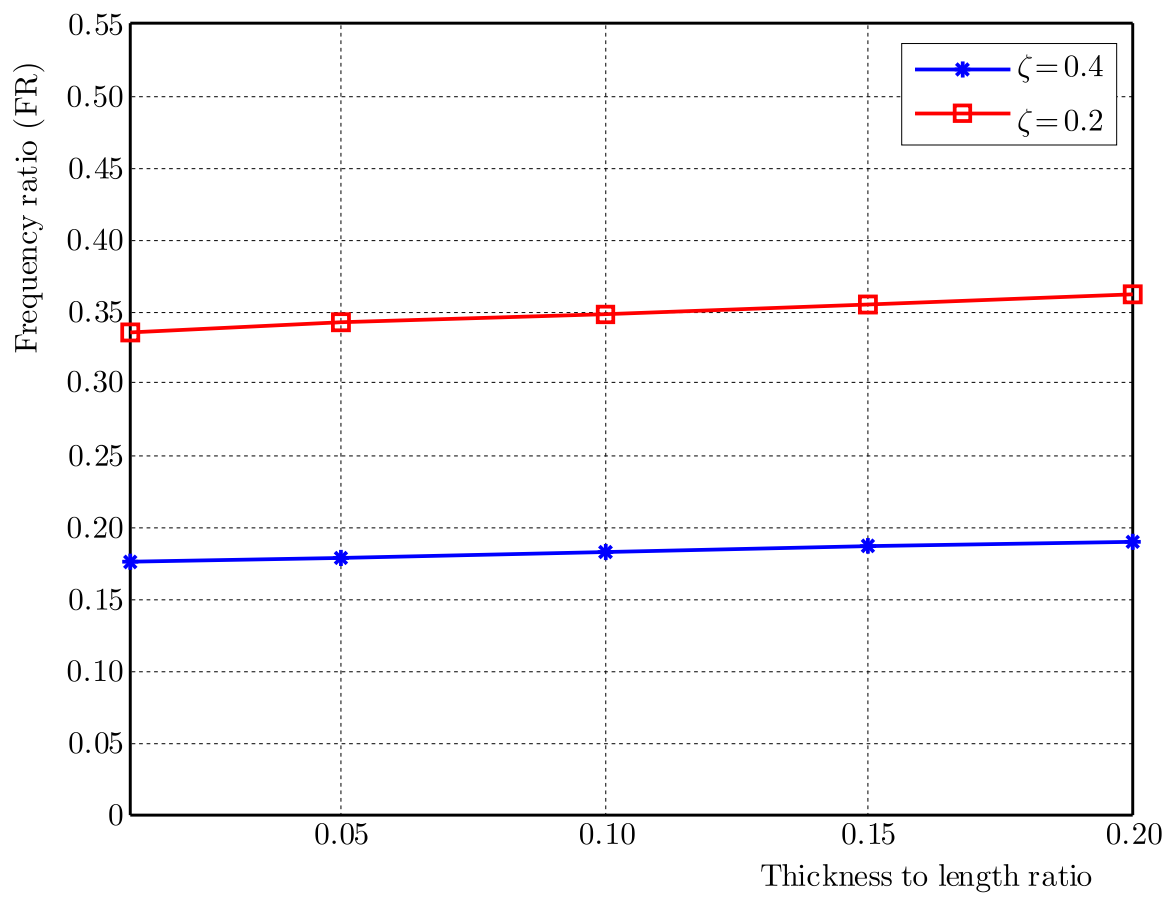

Fig. 2. Variations of the frequency ratio with the thickness to length ratio for SCSC $(\eta=0.5$, $m=1, n=2)$

Table 1. Variations of the non-dimensional frequency $\beta$ and the frequency ratio (FR) for different modes, nonlocal parameters and thickness to length ratios in the case of SCSC boundary condition, $\eta=0.5$ using the nonlocal first-order shear deformation plate theory (Hosseini-Hashemi et al., 2013)

\begin{tabular}{|c|c|c|c|c|}
\hline \multirow{3}{*}{$\delta=h / a$} & \multicolumn{4}{|c|}{$\zeta$} \\
\hline & 0 & 0.2 & 0.4 & 0.6 \\
\hline & \multicolumn{4}{|c|}{$(m=1, n=1)$} \\
\hline 0.01 & $94.9657(1)$ & $51.6164(0.5435)$ & $29.1845(0.3073)$ & $19.9751(0.2103)$ \\
\hline 0.2 & $52.1283(1)$ & $29.5130(0.5662)$ & $16.8392(0.3230)$ & $11.5435(0.2214)$ \\
\hline \multirow[t]{2}{*}{ Diff(FR)\% } & 0 & 4.2 & 5.1 & 5.3 \\
\hline & \multicolumn{4}{|c|}{$(m=1, n=2)$} \\
\hline 0.01 & $252.3968(1)$ & $84.7129(0.3356)$ & $44.2219(0.1752)$ & $29.7293(0.1178)$ \\
\hline 0.2 & $102.7371(1)$ & $37.1430(0.3615)$ & $19.5190(0.1900)$ & $13.1390(0.1279)$ \\
\hline Diff(FR)\% & 0 & 7.7 & 8.4 & 8.6 \\
\hline
\end{tabular}

at all. Note that our reported calculation and conclusion can also be checked and verified by using their reported frequency values from their Table 10 (Hosseini-Hashemi et al., 2015) and recalculating the frequency ratios for all the mentioned boundary conditions.

Table 3 presents the fundamental frequency ratio (FR) for different nonlocal parameters and thickness to length ratios using Reddy's nonlocal third-order shear deformation plate theory by Hosseini-Hashemi et al. (2015) in the case of SSSS boundary condition for three different aspect ratios $a / b=0.5,1,2$. It is seen from Table 3 that the frequency ratio increases with an increase in the thickness to length ratio for a fixed value of the nonlocal parameter. As the nonlocal parameter $\zeta=\sqrt{\mu} / a$ increases, the difference in the fundamental frequency ratio increases and it reaches about $0.7 \%$ in the case of $\zeta=0.6, a / b=0.5$, which is very small and can be neglected for the considered parameters. 
Table 2. Variations of the fundamental frequency $\beta$ and frequency ratio (FR) for different aspect ratios, nonlocal parameters and thickness to length ratios in the case of SCSC boundary condition using the nonlocal third-order shear deformation plate theory (Hosseini-Hashemi et al., 2015)

\begin{tabular}{|c|c|c|c|c|}
\hline \multirow{3}{*}{$h / a$} & \multicolumn{4}{|c|}{$\zeta=\sqrt{\mu} / a$} \\
\hline & 0 & 0.2 & 0.4 & 0.6 \\
\hline & \multicolumn{4}{|c|}{$a / b=0.5$} \\
\hline$\overline{0.05}$ & "88.5692(1.0000) & 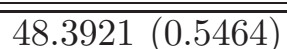 & $=27.3718(0.3090)$ & 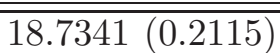 \\
\hline 0.1 & $75.2832(1.0000)$ & $41.6212(0.5529)$ & $23.5827(0.3133)$ & $16.1444(0.2144)$ \\
\hline 0.2 & $53.1087(1.0000)$ & $30.1629(0.5679)$ & $17.1903(0.3237)$ & $11.7824(0.2219)$ \\
\hline \multirow{2}{*}{ Diff(FR)\% } & 0.0 & 3.9 & 4.7 & 4.9 \\
\hline & \multicolumn{4}{|c|}{$a / b=1$} \\
\hline 0.05 & $28.3174(1.0000)$ & $20.6724(0.7300)$ & "13.3181 (0.4703) & $99.4734(0.3345)$ \\
\hline 0.1 & $26.7084(1.0000)$ & $19.5537(0.7321)$ & $12.6161(0.4724)$ & $8.9777(0.3361)$ \\
\hline 0.2 & $22.5355(1.0000)$ & $16.6210(0.7375)$ & $10.7703(0.4779)$ & $7.6740(0.3405)$ \\
\hline \multirow[t]{2}{*}{ Diff(FR)\% } & 0.0 & 1.0 & 1.6 & 1.8 \\
\hline & \multicolumn{4}{|c|}{$a / b=2$} \\
\hline 0.05 & $13.5772(1.0000)$ & $111.0475(0.8137)$ & $\overline{77.7813(0.5731)}$ & $5.7357(0.4224)$ \\
\hline 0.1 & $13.2747(1.0000)$ & $10.8073(0.8141)$ & $7.6159(0.5737)$ & $5.6149(0.4230)$ \\
\hline 0.2 & $12.2939(1.0000)$ & $10.0250(0.8154)$ & $7.0754(0.5755)$ & $5.2198(0.4246)$ \\
\hline Diff(FR)\% & 0.0 & 0.2 & 0.4 & 0.5 \\
\hline
\end{tabular}

Table 3. Variations of the fundamental frequency $\beta$ and frequency ratio (FR) for different aspect ratios, nonlocal parameters and thickness to length ratios in the case of SSSS boundary condition using the nonlocal third-order shear deformation plate theory (Hosseini-Hashemi et al., 2015)

\begin{tabular}{|c|c|c|c|c|}
\hline \multirow{3}{*}{$h / a$} & \multicolumn{4}{|c|}{$\zeta=\sqrt{\mu} / a$} \\
\hline & 0 & 0.2 & 0.4 & 0.6 \\
\hline & \multicolumn{4}{|c|}{$a / b=0.5$} \\
\hline 0.05 & $48.2699(1.0000)$ & $27.9996(0.5801)$ & $16.1910(0.3354)$ & $11.1480(0.2310)$ \\
\hline 0.1 & $45.4869(1.0000)$ & $26.4101(0.5806)$ & $15.2767(0.3358)$ & $10.5194(0.2313)$ \\
\hline 0.2 & $38.1883(1.0000)$ & $22.2526(0.5827)$ & $12.8876(0.3375)$ & $8.8772(0.2325)$ \\
\hline \multirow[t]{2}{*}{ Diff(FR)\% } & 0.0 & 0.4 & 0.6 & 0.65 \\
\hline & \multicolumn{4}{|c|}{$a / b=1$} \\
\hline 0.05 & $19.5625(1.0000)$ & $14.6247(0.7476)$ & $9.5947(0.4905)$ & $6.8721(0.3513)$ \\
\hline 0.1 & $19.0653(1.0000)$ & $14.2566(0.7478)$ & $9.3550(0.4907)$ & $6.7009(0.3515)$ \\
\hline 0.2 & $17.4523(1.0000)$ & $13.0634(0.7485)$ & $8.5780(0.4915)$ & $6.1460(0.3522)$ \\
\hline \multirow[t]{2}{*}{ Diff(FR)\% } & 0.0 & 0.12 & 0.20 & 0.25 \\
\hline & \multicolumn{4}{|c|}{$a / b=2$} \\
\hline 0.05 & $12.2675(1.0000)$ & $10.0386(0.8183)$ & $7.1142(0.5799)$ & $5.2595(0.4287)$ \\
\hline 0.1 & $12.0675(1.0000)$ & $9.8761(0.8184)$ & $6.9999(0.5801)$ & $5.1753(0.4289)$ \\
\hline 0.2 & $11.3717(1.0000)$ & $9.3111(0.8188)$ & $6.6025(0.5806)$ & $4.8826(0.4294)$ \\
\hline Diff(FR)\% & 0.0 & 0.06 & 0.12 & 0.16 \\
\hline
\end{tabular}


It can be deduced from Tables 1-3 that the frequency ratio is indeed dependent on the thickness to length ratio. It is also observed from Tables 2 and 3 that the frequency ratio difference is medium for the plate aspect ratio of $a / b=1$ while the difference is sever and significant up to $5 \%$ for the lower aspect ratio $a / b=0.5$. It is also noticeable that the frequency ratio difference is also dependent on the combination of the boundary conditions by comparing Tables 2 and 3. The observed difference is significant for SCSC boundary condition as presented in Table 2 while the thickness to length ratio has the least effect on the frequency ratio for SSSS boundary condition as presented in Table 3 .

\subsection{Nanobeam results}

In order to detect whether the mentioned conclusions about nanoplates are valid or not for other nanostructures, the process was repeated for nanobeams using the nonlocal first order shear deformation, namely the Timoshenko beam theory (Bahrami and Teimourian, 2016; Reddy, 2007).

Tables 4 and 5 present the frequency ratio (FR) for different modes, nonlocal parameters, and thickness to length ratios using the nonlocal first-order shear deformation theory, namely the Timoshenko beam theory by Reddy (2007) in the cases of CC and SS nanobeams, respectively. The same mentioned conclusions about nanoplates can also be detected via Tables 4 and 5 for the nanobeams as follows:

- The frequency ratio increases with an increase in the thickness to length ratio.

- As the nonlocal parameter increases, the percentage difference in the frequency ratio increases.

- The frequency ratio difference increases as the mode number increases.

- The effect of thickness to length ratio on the frequency ratio is significant for clampedclamped (CC) nanobeams while it has the least effect for simply supported (SS) nanobeams.

Table 4. Variations of the non-dimensional frequency $\sqrt{\beta}$ and the frequency ratio (FR) for different modes, nonlocal parameters and thickness to length ratios in the case of CC nanobeam using the nonlocal first-order shear deformation theory, namely the Timoshenko beam theory (Bahrami and Teimourian, 2016; Reddy, 2007), $L=10 \mathrm{~nm}, E=5.5 \mathrm{TPa}, \nu=0.19, k_{s}=0.563$

\begin{tabular}{|c|c|c|c|c|}
\hline \multirow{3}{*}{$\delta=h / a$} & \multicolumn{4}{|c|}{$\zeta=\sqrt{\mu} / L$} \\
\hline & 0 & 0.2 & 0.4 & 0.6 \\
\hline & \multicolumn{4}{|c|}{ Mode 1} \\
\hline 0.01 & $4.7279(1.0000)$ & $4.2748(0.9041)$ & $3.5908(0.7594)$ & $3.0824(0.6519)$ \\
\hline 0.1 & $4.5348(1.0000)$ & $4.1097(0.9062)$ & $3.4566(0.7622)$ & $2.9675(0.6543)$ \\
\hline$\overline{0.2}$ & $4.1254(1.0000)$ & $3.7559(0.9104)$ & $3.1711(0.7686)$ & $2.7249(0.6605)$ \\
\hline$\overline{0.3}$ & $3.7150(1.0000)$ & $3.3950(0.9138)$ & $2.8792(0.7750)$ & $2.4784(0.6671)$ \\
\hline \multirow[t]{2}{*}{ Diff(FR)\% } & 0.0 & 1.1 & 2.0 & 2.3 \\
\hline & \multicolumn{4}{|c|}{ Mode 2} \\
\hline$\overline{0.01}$ & $7.8449(1.0000)$ & $6.0294(0.7685)$ & $4.5934(0.5855)$ & $3.8129(0.4860)$ \\
\hline 0.1 & $7.1985(1.0000)$ & $5.5728(0.7741)$ & $4.2523(0.5907)$ & $3.5307(0.4904)$ \\
\hline 0.2 & $6.1616(1.0000)$ & $4.8236(0.7828)$ & $3.6959(0.5998)$ & $3.0716(0.4985)$ \\
\hline 0.3 & $5.3496(1.0000)$ & $4.2180(0.7884)$ & $3.2437(0.6063)$ & $2.6987(0.5044)$ \\
\hline Diff(FR)\% & 0.0 & 2.6 & 3.5 & 3.8 \\
\hline
\end{tabular}


Table 5. Variations of the non-dimensional frequency $\sqrt{\beta}$ and the frequency ratio (FR) for different modes, nonlocal parameters and thickness to length ratios in the case of SS nanobeam using the nonlocal first-order shear deformation theory, namely the Timoshenko beam theory (Bahrami and Teimourian, 2016; Reddy, 2007), $L=10 \mathrm{~nm}, E=5.5 \mathrm{TPa}, \nu=0.19, k_{s}=0.563$

\begin{tabular}{|c|c|c|c|c|}
\hline \multirow{3}{*}{$\delta=h / a$} & \multicolumn{4}{|c|}{$\zeta=\sqrt{\mu} / L$} \\
\hline & 0 & 0.2 & 0.4 & 0.6 \\
\hline & \multicolumn{4}{|c|}{ Mode 1} \\
\hline$\overline{0.01}$ & $3.1413(1.0000)$ & $2.8905(0.9201)$ & $2.4788(0.7891)$ & $2.1504(0.6845)$ \\
\hline 0.1 & $3.1089(1.0000)$ & $2.8608(0.9201)$ & $2.4532(0.7891)$ & $2.1283(0.6845)$ \\
\hline 0.2 & $3.0219(1.0000)$ & $2.7807(0.9201)$ & $2.3846(0.7891)$ & $2.0688(0.6845)$ \\
\hline$\overline{0.3}$ & $2.9038(1.0000)$ & $2.6720(0.9201)$ & $2.2914(0.7891)$ & $1.9879(0.6845)$ \\
\hline \multirow[t]{2}{*}{ Diff(FR)\% } & 0.00 & 0.00 & 0.00 & 0.00 \\
\hline & \multicolumn{4}{|c|}{ Mode 2} \\
\hline 0.01 & $6.2805(1.0000)$ & $4 \bar{c} 4.9559(0.7890)$ & $3.8187(0.6080)$ & $3.1801(0.5063)$ \\
\hline 0.1 & $6.0439(1.0000)$ & $4.7692(0.7890)$ & $3.6748 \mathrm{n}(0.6080)$ & $3.0603(0.5063)$ \\
\hline 0.2 & $5.5494(1.0000)$ & $4.3790(0.7890)$ & $3.3742(0.6080)$ & $2.8100(0.5063)$ \\
\hline 0.3 & $5.0518(1.0000)$ & $3.9864(0.7890)$ & $3.0717(0.6080)$ & $2.5580(0.5063)$ \\
\hline Diff(FR)\% & 0.00 & 0.00 & 0.00 & 0.00 \\
\hline
\end{tabular}

\section{Conclusion}

The main intention of this communication is to correct the misunderstanding among researchers regarding the true effect of the thickness to length ratio on the frequency ratio of nanobeams and nanoplates using Eringen's nonlocal theory. It was found that the frequency ratio is indeed dependent on the thickness to length ratio, and its variation with respect to the thickness to length ratio is highly dependent on four parameters, namely: 1) the mode number, 2) combination of boundary conditions, 3) plate aspect ratio, and 4) the nonlocal parameter. The following conclusions were drawn as follows:

- The frequency ratio increases with an increase in the thickness to length ratio, and the percentage difference in the frequency ratio is more significant for a higher nonlocal parameter.

- The frequency ratio decreases with an increase in the nonlocal parameter.

- The frequency ratio difference increases as the mode number increases.

- The frequency ratio difference is more significant for a lower aspect ratio of the nanoplate.

- The observed frequency ratio difference is significant for clamped-clamped (CC) nanobeams while it is almost negligible for simply supported (SS) nanobeams.

- The observed frequency ratio difference is significant for a (SCSC) nanoplate while it is almost negligible for fully the simply supported (SSSS) nanoplate.

- The effect of the thickness to length ratio on the frequency ratio is more significant for nanoplates in comparison to nanobeams.

The authors hope that this communication corrects the misunderstanding regarding this issue. The authors also hope that this communication can motivate other researchers to investigate similar problems with other nonlocal theories in the future.

\section{References}

1. Aghababaei R., Reddy J., 2009, Nonlocal third-order shear deformation plate theory with application to bending and vibration of plates, Journal of Sound and Vibration, 326, 277-289 
2. Arefi M., Zamani M.H., Kiani M., 2018, Size-dependent free vibration analysis of three-layered exponentially graded nanoplate with piezomagnetic face-sheets resting on Pasternak's foundation, Journal of Intelligent Material Systems and Structures, 29, 5, 774-786

3. Arefi M., Zenkour A.M., 2016, Employing sinusoidal shear deformation plate theory for transient analysis of three layers sandwich nanoplate integrated with piezo-magnetic face-sheets, Smart Materials and Structures, 25, 11, 115040

4. Arefi M., Zenkour A.M., 2017a, Nonlocal electro-thermo-mechanical analysis of a sandwich nanoplate containing a Kelvin-Voigt viscoelastic nanoplate and two piezoelectric layers, Acta Mechanica, 228, 475-493

5. Arefi M., Zenkour A.M., 2017b, Size-dependent free vibration and dynamic analyses of piezoelectro-magnetic sandwich nanoplates resting on viscoelastic foundation, Physica B: Condensed Matter, 521, 188-197

6. Arefi M., Zenkour A.M., 2017c, Size-dependent vibration and bending analyses of the piezomagnetic three-layer nanobeams, Applied Physics A, 123, 3, 202

7. Aydogdu M., 2009, A general nonlocal beam theory: its application to nanobeam bending, buckling and vibration, Physica E: Low-dimensional Systems and Nanostructures, 41, 1651-1655

8. BAhrami A., 2017a, A wave-based computational method for free vibration, wave power transmission and reflection in multi-cracked nanobeams, Composites Part B: Engineering, 120, 168-181

9. Bahrami A., 2017b, Free vibration, wave power transmission and reflection in multi-cracked nanorods, Composites Part B: Engineering, 127, 53-62

10. Bahrami A., Ilkhani M.R., Bahrami M.N., 2015, Wave propagation technique for free vibration analysis of annular circular and sectorial membranes, Journal of Vibration and Control, 21, $1866-1872$

11. Bahrami A., Teimourian A., 2015a, Free vibration analysis of composite, circular annular membranes using wave propagation approach, Applied Mathematical Modelling, 39, 4781-4796

12. Bahrami A., Teimourian A., 2015b, Nonlocal scale effects on buckling, vibration and wave reflection in nanobeams via wave propagation approach, Composite Structures, 134, 1061-1075

13. Bahrami A., Teimourian A., 2016, Study on the effect of small scale on the wave reflection in carbon nanotubes using nonlocal Timoshenko beam theory and wave propagation approach, Composites Part B: Engineering, 91, 492-504

14. Bahrami A., Teimourian A., 2017a, Small scale effect on vibration and wave power reflection in circular annular nanoplates, Composites Part B: Engineering, 109, 214-226

15. Bahrami A., Teimourian A., 2017b, Study on vibration, wave reflection and transmission in composite rectangular membranes using wave propagation approach, Meccanica, 52, 231-249

16. Daneshmehr A., Rajabpoor A., Hadi A., 2015, Size dependent free vibration analysis of nanoplates made of functionally graded materials based on nonlocal elasticity theory with high order theories, International Journal of Engineering Science, 95, 23-35

17. Dehrouyeh-Semnani A.M., Bahrami A., 2016, On size-dependent Timoshenko beam element based on modified couple stress theory, International Journal of Engineering Science, 107, 134-148

18. Eltaher M., Emam S.A., Mahmoud F., 2012, Free vibration analysis of functionally graded size-dependent nanobeams, Applied Mathematics and Computation, 218, 7406-7420

19. ERINGEN A.C., 1972, Theory of micromorphic materials with memory, International Journal of Engineering Science, 10, 623-641

20. Eringen A.C., 2002, Nonlocal Continuum Field Theories, Springer Science \& Business Media

21. Hosseini-Hashemi S., Kermajani M., Nazemnezhad R., 2015, An analytical study on the buckling and free vibration of rectangular nanoplates using nonlocal third-order shear deformation plate theory, European Journal of Mechanics-A/Solids, 51, 29-43 
22. Hosseini-Hashemi S., Zare M., Nazemnezhad R., 2013, An exact analytical approach for free vibration of Mindlin rectangular nano-plates via nonlocal elasticity, Composite Structures, 100, 290-299

23. Ilkhani M.R., Bahrami A., Hosseini-Hashemi S.H., 2016, Free vibrations of thin rectangular nano-plates using wave propagation approach, Applied Mathematical Modelling, 40, 1287-1299

24. Kahrobaiyan M.H., Asghari M., Rahaeifard M., Ahmadian M.T., 2010, Investigation of the size-dependent dynamic characteristics of atomic force microscope microcantilevers based on the modified couple stress theory, International Journal of Engineering Science, 48, 1985-1994

25. Lim C.W., Zhang G., Reddy J.N., 2015, A higher-order nonlocal elasticity and strain gradient theory and its applications in wave propagation, Journal of the Mechanics and Physics of Solids, 78, 298-313

26. Mindlin R.D., 1964, Micro-structure in linear elasticity, Archive for Rational Mechanics and Analysis, 16, 51-78

27. Mindlin R.D., Tiersten H.F., 1962, Effects of couple-stresses in linear elasticity, Archive for Rational Mechanics and Analysis, 11, 415-448

28. Natarajan S., Chakraborty S., Thangavel M., Bordas S., Rabczuk T., 2012, Sizedependent free flexural vibration behavior of functionally graded nanoplates, Computational Materials Science, 65, 74-80

29. Rahmani O., Pedram O., 2014, Analysis and modeling the size effect on vibration of functionally graded nanobeams based on nonlocal Timoshenko beam theory, International Journal of Engineering Science, 77, 55-70

30. REDDy J., 2007, Nonlocal theories for bending, buckling and vibration of beams, International Journal of Engineering Science, 45, 288-307

31. Sarayi S.M.M.J., Bahrami A., Bahrami M.N., 2018. Free vibration and wave power reflection in Mindlin rectangular plates via exact wave propagation approach, Composites Part B: Engineering, 144, 195-205

32. Thai H.T., 2012, A nonlocal beam theory for bending, buckling, and vibration of nanobeams, International Journal of Engineering Science, 52, 56-64

33. Yang F., Chong A.C.M., LAm D.C.C., Tong P., 2002. Couple stress based strain gradient theory for elasticity, International Journal of Solids and Structures, 39, 2731-2743

34. Zargaripoor A., Bahrami A., 2018. Free vibration and buckling analysis of third-order shear deformation plate theory using exact wave propagation approach, Journal of Computational Applied Mechanics, 49, 102-124

35. Zenkour A.M., Arefi M., 2017. Nonlocal transient electrothermomechanical vibration and bending analysis of a functionally graded piezoelectric single-layered nanosheet rest on visco-Pasternak foundation, Journal of Thermal Stresses, 40, 2, 167-184

36. Zhang J., Fu Y., 2012, Pull-in analysis of electrically actuated viscoelastic microbeams based on a modified couple stress theory, Meccanica, 47, 1649-1658 